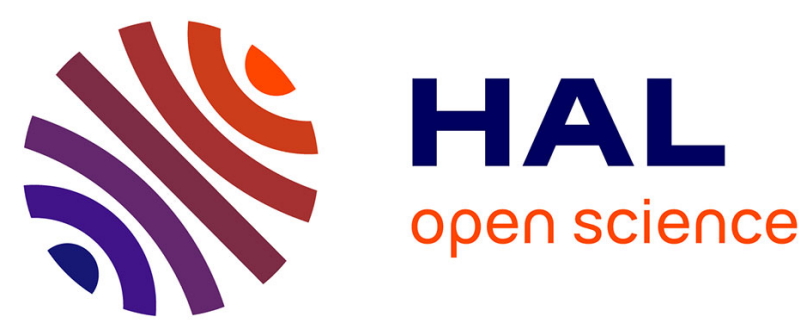

\title{
PADS 2-06 - Paludisme et orpaillage en Guyane: une situation alarmante mais hétérogène
}

\author{
Maylis Douine, L. Musset, F. Corlin, S. Pelleau, L. Mutricy, D. Blanchet, \\ Félix Djossou, H. Hiwat, Magalie Pierre Demar, Mathieu Nacher
}

\section{- To cite this version:}

Maylis Douine, L. Musset, F. Corlin, S. Pelleau, L. Mutricy, et al.. PADS 2-06 - Paludisme et orpaillage en Guyane: une situation alarmante mais hétérogène. 17es Journées Nationales d'Infectiologie, Jun 2016, Lille, France. Médecine et maladies infectieuses 46 (2016) 93-94, 46 (4), pp.93 - 94 . inserm01410986

\section{HAL Id: inserm-01410986 https://www.hal.inserm.fr/inserm-01410986}

Submitted on 6 Dec 2016

HAL is a multi-disciplinary open access archive for the deposit and dissemination of scientific research documents, whether they are published or not. The documents may come from teaching and research institutions in France or abroad, or from public or private research centers.
L'archive ouverte pluridisciplinaire HAL, est destinée au dépôt et à la diffusion de documents scientifiques de niveau recherche, publiés ou non, émanant des établissements d'enseignement et de recherche français ou étrangers, des laboratoires publics ou privés. 


\section{PADS 2-03}

\section{Uvéite syphilitique : étude rétrospective tricentrique de 64 patients}

F. Hoogewood (1), L. Frumholtz (1), P. Loubet (1), C. Charlier (2), P. Blanche (1), O. Launay (1), O. Lortholary (2), N. Dupin (1), A. Brezin (1), M. Groh (1)

(1) CHU Cochin, Paris,

(2) CHU Necker, Paris.

Introduction Par analogie avec la neurosyphilis, le traitement (TTT) de l'uvéite syphilitique est basé sur la pénicilline G (péni G) intraveineuse. Des données préliminaires suggèrent que les cephalosporines de $3^{\mathrm{e}}$ génération $(\mathrm{C} 3 \mathrm{G})$ et la benzathine pénicilline pourraient être efficaces.

Matériels et méthodes Les patients traités entre 2004 et 2014 dans 3 CHU pour une uvéite syphilitique ont été inclus. Les données ont été recueillies à J0, J7 et M1 après début du TTT. A J7, l'amélioration clinique était définie comme une diminution $\geq 2+$ de l'inflammation en chambre antérieure (CA), dans le vitré, et une diminution de la taille du foyer rétinien. A M1, la guérison était définie comme l'absence de cellules en CA, de hyalite, de foyer actif, et de papillite.

La guérison a été étudiée en fonction du délai entre l'apparition des premiers symptômes et le début du TTT, du type d'atteinte oculaire, du statut sérologique pour le VIH, du caractère anormal ou non de la ponction lombaire (PL), du type de TTT (groupe A : péni G exclusive; groupe $\mathrm{B}: \geq 3$ jours de péni $\mathrm{G}$ relayée par un TTT intramusculaire par $\mathrm{C} 3 \mathrm{G}$ et/ou benzathine pénicilline ; groupe C : TTT intramusculaire ambulatoire exclusif) et de l'évolution à J7. Les facteurs associés à la guérison en analyse univariée (seuil p < 0.2) ont été explorés grâce à une analyse multivariée de type régression logistique pas à pas descendante ajustée sur l'âge et le sexe.

Résultats Soixante-quatre patients (92 yeux) ont été inclus, dont 29 (45\%) étaient séropositifs pour le VIH. L'atteinte ophtalmologique était bilatérale chez $28(44 \%)$ patients, dont $14(50 \%)$ avaient une atteinte asymétrique. Les manifestations oculaires les plus fréquentes étaient la panuvéite et l'uvéopapillite, présentes dans $35(38 \%)$ et $20(22 \%)$ yeux respectivement.

Parmi les $51(80 \%)$ patients ayant un suivi post-TTT $\geq 1$ mois, seuls $27(53 \%)$ étaient guéris à M1. En analyse univariée, le caractère postérieur isolé de l'uvéite et l'amélioration précoce des lésions à $\mathrm{J} 7$ étaient corrélés à la guérison à $\mathrm{M} 1$ ( $\mathrm{p}=0,01$ et $\mathrm{p}=0,001$ respectivement). En analyse multivariée, seule l'amélioration des lésions à $\mathrm{J} 7$ restait corrélée à la guérison (OR 11.2 IC95 \% 2.6-47.9; $\mathrm{p}=0,001$ ).

Conclusion Après un TTT initial par péni G, le relai intramusculaire semble être une alternative au TTT par péni $\mathrm{G}$ exclusive des patients évoluant favorablement à $\mathbf{J} 7$. Ces données pourraient faciliter la prise en charge ambulatoire des patients mais nécessitent d'être confirmées par un essai prospectif randomisé contrôlé.

Aucun lien d'intérêt

\section{PADS 2-04}

Caractéristiques de santé sexuelle des jeunes filles consultant dans un centre de dépistage parisien

N. Valin (1), L. Fonquernie (2), H. Bideault (3), H. Guyon (4), L. MorandJoubert (5), D. Decré (6), C. Gachenot (7), E. Torres (8), P. Girard (9), O. Picard (10)

(1) hôpital Saint-Antoine, Paris.

Introduction Les centres de dépistage anonyme et gratuits (CDAG) ont été transformés en Centre gratuit d'Information de Dépistage et de Diagnostic (CeGIDD) au $1^{\text {er }}$ janvier 2016 avec des missions élargies de prise en charge globale de la santé sexuelle.

Objectif : L'objectif est de décrire les caractéristiques en termes de santé sexuelle de la population des jeunes filles âgées de moins de 25 ans consultant dans un CDAG parisien.

Matériels et méthodes Méthodes : Entre le 01/01/2015 et le 31/12/2015, les données sociodémographiques et cliniques de l'ensemble des jeunes filles de moins de 25 ans consultant au CDAG d'un hôpital parisien ont été analysées à partir du logiciel DiammG ${ }^{\circledR}$.

Résultats Sur l'année 2015, 844 jeunes filles $\leq 25$ ans ont consulté au CDAG. L'âge médian est de 22 ans (de 14 à 25 ans), 733 (87\%) sont d'origine caucasienne. Pour $324(38 \%)$, il s'agit d'un premier recours au dépistage. Près d'un tiers d'entre elles (312) n'a aucune contraception, y compris le préservatif ; $67(8 \%)$ ont déjà eu recours au moins une fois à une interruption volontaire de grossesse ; $110(13 \%)$ ont un antécédent d'IST. Le nombre médian de partenaires dans la dernière année est de 2 . Les résultats du dépistage systématique par autoprélèvement montrent une prévalence de $9 \%(61)$ d'infection à Chlamydia trachomatis et $0.8 \%$ (6) d'infection à Neisseria gonorrhaoe. Plus de la moitié des jeunes filles (51\%) a des anticorps anti HBs négatifs. Aucune sérologie VIH n'est revenue positive dans cette population.

Conclusion L'élargissement des missions des CeGIDD avec un accès facilité aux consultations de gynécologie, à la vaccination contre l'hépatite $\mathrm{B}$ et à la prise en charge de la santé sexuelle globale semble particulièrement intéressant pour cette population jeune dont la couverture vaccinale et le recours à la contraception restent insuffisants.

Aucun lien d'intérêt

\section{PADS 2-05}

Accueil médical des réfugiés syriens et irakiens et pathologies infectieuses

N. Vignier (1), V. Jeannerod (2), V. Pires (3), M. Postal Pâques (3), P. Bouzidi (3), H. Kassem (3), M. Alloujami (3), S. Dimi (3), S. Diamantis (3), F. Dolveck (3)

(1) $\mathrm{CH}$ de Melun, Melun, France,

(2) RVH 77 Sud, Melun, France,

(3) $\mathrm{CH}$ de Melun, Melun, France.

Introduction Suite à la crise Syrienne et l'annonce présidentielle, 88 réfugiés syriens et irakiens ont été accueillis dans une résidence de notre territoire. L'accueil médical de ces réfugiés n'a pas été organisé en amont par les autorités sanitaires.

Matériels et méthodes Un recueil systématique des actions et consultations a été mis en place au cours de cette action. Un codage détaillé des pathologies infectieuses rencontrées a été mis en place.

Résultats Des consultations régulières ont été assurées jusqu'à l'obtention de la CMU par les résidents avec l'aide de médiateurs arabophones. Au total, 101 consultations ont été réalisées en 7 permanences pour 73 patients (79\% d'hommes, $22 \%$ enfants, $58 \%$ adultes de moins de 40 ans). Le premier motif de consultation était les pathologies infectieuses $(37 \%)$, suivi par les problèmes dentaires $(15 \%)$ et les problèmes dermatologiques $(11 \%)$. A noter également des syndromes anxieux post traumatiques en particulier parmi les enfants et un suivi de grossesse non compliqué. Les pathologies infectieuses rencontrées étaient principalement des infections virales aiguës $(71 \%)$ et des infections bactériennes aiguës $(18 \%)$ dont une pneumopathie sévère ayan nécessité une hospitalisation. 24 patients ont été orientés vers une consultation spécialisée grâce au partenariat avec l'hôpital (dentiste, ORL, gynéco, etc.).

Une action de médecine préventive a été organisée avec dépistage de la tuberculose par radio pulmonaire, dépistage du VIH et des hépatites B et $\mathrm{C}$ et mise à jour des vaccinations. Sur 76 personnes dépistées, une radio était anormale et a motivé une hospitalisation qui a permis de mettre en évidence une tuberculose pulmonaire multirésistante EM- culture+ (INH-R, RIF-R, ETH$\mathrm{R})$. Toutes les sérologies virales se sont révélées négatives. 178 vaccins ont été réalisés (principalement mise à jour DTPC et ROR)

Après l'obtention de la CMU, nous avons cessé les consultations mais les réfugiés ont fait l'objet de refus de soins médicaux et dentaire de la part des confrères libéraux du territoire.

Conclusion Les pathologies infectieuses bénignes sont au premier rang des motifs de consultation de réfugiés irakiens et syriens arrivant sur le territoire. Il parait urgent d'anticiper et d'organiser l'accueil médical des réfugiés arrivant en France qu'ils soient en situation régulière ou irrégulière et de lutter contre le refus de soins une fois la couverture maladie acquise.

Aucun lien d'intérêt

\section{PADS 2-06}

Paludisme et orpaillage en Guyane : une situation alarmante mais hétérogène

M. Douine (1), L. Musset (2), F. Corlin (1), S. Pelleau (2), L. Mutricy (1) D. Blanchet (3), F. Djossou (4), H. Hiwat (5), M. Demar (3), M. Nacher (1) (1) Centre d'Investigation Clinique, Centre Hospitalier de Cayenne, Cayenne, (2) Laboratoire de parasitologie, CNR du paludisme, Institut Pasteur de la Guyane, Cayenne,

(3) Laboratoire de Parasitologie-Mycologie, Centre Hospitalier de Cayenne, Cayenne, 
(4) Unité des Maladies Infectieuses et Tropicales, Centre Hospitalier de Cayenne, Cayenne,

(5) Ministry of Health, Malaria Program, Paramaribo, Suriname.

Introduction Le paludisme est en nette diminution en Guyane actuellement. Des foyers de transmission persistent en population autochtone, mais cette pathologie touche aujourd'hui majoritairement les orpailleurs clandestins travaillant en Guyane. Cette étude avait pour objectifs d'évaluer le portage de Plasmodium par bassin d'orpaillage et les connaissances, attitudes et pratiques (CAP) des orpailleurs clandestins vis-à-vis du paludisme.

Matériels et méthodes L'étude a été réalisée de janvier à juin 2015 sur les bases de repli et d'approvisionnement des orpailleurs sur la rive surinamaise du fleuve Maroni. Etaient inclus les orpailleurs travaillant en Guyane, majeurs, et présents sur le site depuis moins de 7 jours. Un test de diagnostic rapide du paludisme était réalisé sur place suivi d'un traitement si nécessaire. Un prélèvement sanguin était réalisé pour recherche de plasmodies par examen microscopique et PCR. Un questionnaire recueillait les données sociodémographiques, de mode de vie sur les sites, ainsi que les données CAP. Les autorisations éthiques et réglementaires ont été obtenues en France et au Suriname.

Résultats 421 personnes ont été incluses dans l'étude, d'âge moyen de 37 ans [30-45], avec $70.6 \%$ d'hommes, en majorité brésiliens (93.8\%). La prévalence de portage de Plasmodium était de $22.3 \%$, dont $84 \%$ étaient asymptomatiques. Après regroupement des 68 sites d'orpaillage de provenance en 10 zones, la prévalence variait de $3.8 \%$ à $46.4 \%$, la région la plus impaludée étant vers Maripa Soula. Lors du dernier épisode de paludisme, $53.7 \%$ des personnes interrogées déclaraient s'être automédiquées, part des dérivés de l'artémisinine dans $90 \%$ des cas. L'observance déclarée était bonne dans $60 \%$ des cas. Après analyse multivariée, le fait d'être en Guyane lors de l'épisode de paludisme était associé à l'automédication $(\mathrm{OR}=27.8$, $95 \%=9.5-81.6$ ). Ceci montre la difficulté d'un accès effectif aux soins en Guyane pour les orpailleurs clandestins.

Conclusion Le paludisme affecte fortement les orpailleurs clandestins en Guyane, conduisant à une probable immunité partielle avec portage asymptomatique de Plasmodium. L'automédication fréquente et incomplète avec des dérivés de l'artémisinine fait craindre l'apparition de résistance. L'hétérogénéité de la prévalence montre que la zone de Maripa Soula doit être une priorité d'action. Mais la forte mobilité des orpailleurs fait craindre une propagation locale et régionale, nécessitant une réponse sanitaire urgente.

Aucun lien d'intérêt

\section{PADS 2-07}

\section{La leptospirose en Guyane française : $1^{\text {re }}$ étude transversale multicen-} trique 2007-2014.

P. Le Turnier (1), L. Epelboin (1), E. Mosnier (1), R. Schaub (1), R. Boukhari (2), A. Berlioz-Arthaud (3), P. Bourhy (4), F. Djossou (1)

(1) CH Andrée Rosemon, Cayenne, Guyane Française,

(2) CH Ouest Guyanais, St Laurent du Maroni, Guyane Française,

(3) Institut Pasteur de Guyane, Cayenne, Guyane Française,

(4) Institut Pasteur, Paris.

Introduction La leptospirose est une zoonose de répartition mondiale avec une incidence en augmentation. Elle représente un problème de santé publique en zone tropicale. L'objectif de notre étude était de faire un état des lieux de la leptospirose en Guyane Française afin d'en améliorer la prise en charge.

Matériels et méthodes Les cas suspects de leptospirose de 2007 à 2014 ont été identifiés rétrospectivement avec les données PMSI et diagnostics biologiques de leptospirose issus de 2 hôpitaux de Guyane. Les cas étaient confirmés par PCR positive (sang, urine, et/ou LCR), séroconversion en Microscopic Agglutination Test (MAT, technique de référence sérologique), avec titre $\geq 1 / 200$ ou séroascension MAT $\geq 4$ fois sur 2 échantillons ou titre MAT $\geq 1 / 400$. Les cas étaient probables si MAT $=1 / 200$ sans séroconversion avec $\operatorname{IgM} \geq 1 / 800$ ou MAT $=1 / 100$ avec séroconversion en IgM ou titre $\operatorname{IgM} \geq 1 / 800$. Les données démographiques, cliniques, radiologiques, biologiques, et évolutives ont été collectées à partir des dossiers médicaux.

Résultats Soixante-douze patients ont répondu aux critères d'inclusion. Le taux d'incidence moyen était de 4,1/100 000 habitants/an. Cinquante-cinq $(76,4 \%)$ cas étaient confirmés et $17(23,6 \%)$ probables. Le sex-ratio était de 6,2. L'âge moyen était 40,4 (extrêmes: 16-82). Fièvre (91,7\%), céphalées (70 \%), asthénie $(61,4 \%)$ et myalgies $(52,9 \%)$ étaient les symptômes les plus fréquemment rapportés. Le dosage initial de CRP était $>50 \mathrm{mg} / 1$ dans $88,9 \%$ des cas. Le cliché thoracique était anormal dans $48,1 \%$ des cas. Soixantedeux $(80,6 \%)$ patients étaient hospitalisés, dont $12(16,7 \%)$ admissions en réanimation. La mortalité hospitalière était de 4,2\% (3 cas). La leptospirose était initialement suspectée par les cliniciens dans $20 \%$ des cas. L'identification des sérogroupes en MAT retrouvait principalement Icterohemorragiae $(19 / 50 ; 38 \%)$, Australis, Ballum, Canicola et Tarassovi $(4 / 50(8 \%)$ pour chacun). Une exposition professionnelle potentielle était rapportée dans 64,6\% (31/48) dont l'orpaillage en forêt (12/48) et les Bâtiment et Travaux Publics (7/48). Un séjour en forêt, un contact avec des rongeurs et une baignade en rivière étaient des facteurs potentiels d'exposition notés dans respectivement 23,13 et 13 cas.

Conclusion Cette première étude multicentrique a révélé une incidence relativement faible de la leptospirose, mais probablement sous-estimée. La diversité des sérogroupes incriminés souligne la question du réservoir animal qui reste à définir. D'autres études sont nécessaires pour mieux évaluer l'incidence, les facteurs pronostics et prédictifs de la leptospirose, face à d'autres pathologies au tableau clinique initial similaire comme les arboviroses.

Aucun lien d'intérêt

\section{PADS 2-08}

Comment vacciner plus de $\mathbf{5 0 0}$ malades à l'hôpital en moins de $\mathbf{2}$ ans et demi ? utilisez votre CVI !

I. Bukreyeva, L. Tzara, O. Derradji, JC. Gagnard, L. Escaut, D. Vittecoq, B. Wyplosz

CHU Bicêtre, Le Kremlin-Bicêtre, France.

Introduction Le parcours de vaccination des malades comprend de nombreuses étapes (prescription, achat du vaccin, conservation à $+4^{\circ} \mathrm{C}$, injection en ville, etc) qui sont autant d'obstacles à l'obtention d'une couverture vaccinale optimale. Les centres de vaccinations internationaux (CVI) présents dans les hôpitaux sont des structures qui pourraient être utilisées pour vacciner les malades consultant leur spécialiste hospitalier.

L'objectif de ce travail est de raccourcir le parcours de vaccination en utilisant le CVI afin d'améliorer la couverture vaccinale des malades consultant dans notre CHU.

Matériels et méthodes Nous avons proposé aux médecins de la policlinique de prescrire sur une ordonnance les vaccins qu'ils jugeaient utiles pour leurs malades (hors vaccins du voyage) puis de les adresser à l'infirmier du CVI pour une vaccination immédiate sans rendez-vous.

Résultats Entre le 01/06/2013 et le 30/11/2015 (soit 2,5 ans), les médecins de la policlinique nous ont adressé 528 personnes $(\mathrm{H} / \mathrm{F}: 1,2)$ qui ont reçu 895 vaccins (1,7/personnes) au cours de 751 visites (1,4/personnes). Ils étaient suivis dans les services de maladies infectieuses $(34 \%)$, médecine interne (23\%), centre de précarité $(16 \%)$, centre de drépanocytose $(10 \%)$ et d'autres services $(17 \%)$

Nous avons vaccinés une grande majorité d'immunodéprimés $(\mathrm{N}=344$, $65 \%$ ) dont 186 malades vivant avec le VIH (54\%), 51 drépanocytaires (22\%), 29 transplantés ( $8 \%$ ) et $78(23 \%)$ autres maladies (auto-immunes, diabète traité à l'insuline, insuffisance rénale terminale, etc.). Les autres personnes $(\mathrm{N}=184,35 \%)$ venaient principalement de la consultation de précarité.

Les vaccins suivants ont été administrés : pneumocoques $(\mathrm{N}=298,33 \%)$, diphtérie-tétanos-poliomyélite $+/-$ coqueluche $(\mathrm{N}=200,22 \%)$, grippe $(\mathrm{N}=150,17 \%)$, hépatite $\mathrm{B}(\mathrm{N}=127,14 \%)$, hépatite $\mathrm{A}(\mathrm{N}=74,8 \%)$, méningocoque $\mathrm{C}(\mathrm{N}=14,2 \%)$, autres (méningocoques ACYW, Haemophilus influenzae $b$, ROR) $(\mathrm{N}=32,4 \%)$. A noter, que $86(41 \%)$ des 212 vaccinés contre les pneumocoques sont revenus au CVI pour recevoir une $2^{\mathrm{e}}$ dose (conjugué/non-conjugué).

Conclusion Notre offre de vaccination sans rendez-vous en sortie de consultation a été adoptée par les médecins de la policlinique et par les malades qui reviennent, le cas échéant, faire des injections complémentaires. Elle regroupe en une fois plusieurs étapes du parcours de vaccination : délivrance, administration, inscription sur le carnet de vaccination et programmation des rappels. Cette offre de soins permet d'améliorer la couverture vaccinale des malades à l'hôpital. Nous présenterons les résultats obtenus à 3 ans.

Liens d'intérêts déclarés :

BW participe à un board de vaccination pour Pfizer 